\title{
T-duality of axial and vector dyonic integrable models
}

\author{
J.F. Gomes ${ }^{\dagger}$, E. P. Gueuvoghlanian ${ }^{\dagger}$, G.M. Sotkov ${ }^{\dagger}$ and A.H. Zimerman ${ }^{\dagger}$ \\ ${ }^{\dagger}$ Instituto de Física Teórica - IFT/UNESP \\ Rua Pamplona 145 \\ 01405-900, São Paulo - SP, Brazil \\ jfg@ift.unesp.br, gueuvogh@ift.unesp.br, sotkov@ift.unesp.br, zimerman@ift.unesp.br
}

\begin{abstract}
A general construction of affine Non Abelian (NA) - Toda models in terms of axial and vector gauged two loop WZNW model is discussed. They represent integrable perturbations of the conformal $\sigma$-models (with tachyons included) describing (charged) black hole type string backgrounds. We study the off-critical T-duality between certain families of axial and vector type of integrable models for the case of affine NA- Toda theories with one global U(1) symmetry. In particular we find the Lie algebraic condition defining a subclass of T-selfdual torsionless NA Toda models and their zero curvature representation.
\end{abstract}




\section{Introduction}

Two dimensional integrable models represent an important laboratory for testing new ideas and developing new methods for constructing exact solutions as well as for the nonperturbative quantization of 4-D non-abelian gauge theories, gravity and string theory. Among the numerous techniques for constructing 2-D integrable models and their solutions [1], [2], the hamiltonian reduction of the WZNW model (or equivalently the gauged WZNW) associated to a finite dimensional Lie algebra $\mathcal{G}$ has provided an universal and simple method for deriving the equations of motion (or action ) of 2-d integrable models. In particular, the conformal Toda (CT) models were constructed by implementing a consistent set of constraints on the WZNW currents [3]. The method was subsequently extended to infinite dimensional affine algebras ( denoted here by $\hat{\mathcal{G}}$ ), leading to WZNW currents satisfying the so called two loop current algebra [4]. By further imposing an infinite number of suitable constraints the conformal affine Toda (CAT) models were constructed [5]. The power of such method was demonstrated in constructing (multi) soliton solutions of the abelian affine Toda models [5] and certain nonsingular nonabelian (NA) affine Toda models [6].

The present paper is devoted to the systematic construction of a new family of axial and vector affine NA Toda integrable models (associated to an affine Kac-Moody algebra of rank $\mathrm{r}$ - $\hat{\mathcal{G}}_{r}$ ) having one global U(1) symmetry. They represent appropriate integrable perturbations of the conformal $\sigma$-models (with tachyons and dilaton included) describing strings on curved backgrounds of black hole type (see ref.[7], [8]). An important feature of these integrable models is that they admit $\mathrm{U}(1)$-charged topological solitons for imaginary coupling constant [9]. With topological $\theta$-term added to their actions, the one soliton spectrum manifests properties quite similar to the dyons of 4-D Yang-Mills -Higgs model namely, their electric charges get contributions from the magnetic (topological) one. The conformal limits (without the tachyonic terms) of axial and vector models in consideration, are known to be T-dual to each other, having $O(r, r \mid Z)$ as T-duality group (see for example [10]). The natural question arises whether one can extend the critical (i.e. conformal) T-duality transformations to the corresponding integrable model and which is the off-critical T-duality group. The problem of non conformal (i.e. off critical ) T-duality was first addressed in [11] in the context of the principal chiral $\sigma$-models. For more general discussion of T-duality as canonical transformations of conformal and non-conformal 2-d models see [12]. It turns out that axial and vector models similarly to their conformal $\sigma$ - models counterparts are forming again T-dual pairs. The critical T-duality group is however broken to $O(1,1 \mid Z)$ for the family of dyonic integrable models under investigation.

As in the conformal case the subclass of T-selfdual models is of particular interest. We first investigate the (torsionless) T-selfdual conformal $\mathcal{G}_{r}$-NA Toda models obtained from the conformal $\sigma$-models (associated to finite dimensional Lie algebras $\mathcal{G}_{r}$ ) by adding all the possible marginal operators. As it shown in Sect.4.1, T-seldual conformal NA -Toda models exist only for orthogonal algebras $\mathcal{G}_{r}=B_{r}=S O(2 r+1)$ and for specific choice of the form of the marginal operators only. The axial and vector dyonic models can be obtained by adding certain integrable relevant operators to the conformal $\mathcal{G}_{r}$-NA Toda actions. As is well known [2], [6] the algebraic structure underlying these nonconformal affine $\hat{\mathcal{G}}_{r}$-NA Toda models is encodded in the choice of grading operator $\mathrm{Q}$ for $\hat{\mathcal{G}}_{r}$ and in the form of the grade \pm 1 constant 
elements $\epsilon_{ \pm}$(which characterizes the potential). Addressing once more the question of the T-selfdual (torsionless) members of the considered family of integrable models, we derive the Lie algebraic condition (i.e. all the possible choices of the algebraic data $-\hat{\mathcal{G}}_{r}, Q, \epsilon_{ \pm}$) that gives rise to T-selfdual models. It shown in sect. 4.2 and 4.3 that such subclass of models exist only for the following three affine Kac-Moody algebras: $B_{r}^{(1)}, A_{2 r}^{(2)}$ and $D_{r+1}^{(2)}$ when the grading operator $\mathrm{Q}$ and the $\epsilon_{ \pm}$'s are appropriately choosen.

An interesting byproduct of our algebraic T-selfduality condition is that the above three families of torsionless models exactly reproduce the Fateev's integrable models [14]. Our construction also provides a simple and systematic proof of their classical integrability. It is worthwhile to mention the following simple form of our T-selfduality condition: while the generic axial and vector models ( with one global $\mathrm{U}(1)$ symmetry) are characterized by the fact that their physical fields $g_{0}^{f}$ lie in the coset $\mathcal{G}_{0} / \mathcal{G}_{0}^{0}=\frac{S L(2) \otimes U(1)^{\text {rank } \mathcal{G}-1}}{U(1)}$, the corresponding cosets for the T-selfdual models are of the particular form $\mathcal{G}_{0} / \mathcal{G}_{0}^{0}=\frac{S L(2)}{U(1)} \otimes U(1)^{\text {rank } \mathcal{G}^{-1}}$.

This paper is organized as follows. Sect.2 contains the functional integral derivation of the effective actions for generic conformal,affine and conformal affine NA -Toda theories. In the particular case when these models manifest $\mathcal{G}_{0}^{0}=\mathrm{U}(1)$ gauge symmetry, the actions for the corresponding singular affine NA Toda models of axial and vector type are obtained. Two particular examples based on the affine algebras $A_{r}^{(1)}$ and $B_{r}^{(1)}$ are presented. Sect.3 is devoted to the analysis of the abelian off-critical T-duality relating the axial and vector type of models. We derive the Lie algebraic condition defining the family of $T$-selfdual torsionless singular affine NA Toda theories in Sect.4. In Sect.5 we present the zero curvature representations of all IM's in consideration.

\section{Gauged WZNW Construction of NA Toda Models}

The generic conformal (or affine) $G_{r}$ (or $\hat{G}_{r}$ ) - NA - Toda models are classified according to a $\mathcal{G}_{0} \subset \mathcal{G}$ embedding induced by the grading operator $Q$ [2], which defines a specific decomposition of the corresponding finite $\mathcal{G}_{r}$ or infinite $\left(\hat{\mathcal{G}}_{r}\right)$ dimensional Lie algebras $\mathcal{G}=$ $\oplus_{i} \mathcal{G}_{i}$ where $\left[Q, \mathcal{G}_{i}\right]=i \mathcal{G}_{i}$ and $\left[\mathcal{G}_{i}, \mathcal{G}_{j}\right] \subset \mathcal{G}_{i+j}$. The group element $g$ can then be written in terms of the Gauss decomposition as

$$
g=N B M
$$

where $N=\exp \mathcal{G}_{<} \in H_{-}, B=\exp \mathcal{G}_{0}$ and $M=\exp \mathcal{G}_{>} \in H_{+}$. The physical fields $B$ lie in the zero grade subgroup $\mathcal{G}_{0}$ and the models we seek correspond to the coset $H_{-} \backslash G / H_{+}$.

For consistency with the Hamiltonian reduction formalism, the phase space of the Ginvariant WZNW model is reduced by specifying the constant generators $\epsilon_{ \pm}$of grade \pm 1 . In order to derive an action for $B \in \mathcal{G}_{0}$, invariant under

$$
g \longrightarrow g^{\prime}=\alpha_{-} g \alpha_{+},
$$

where $\alpha_{ \pm}(z, \bar{z}) \in H_{ \pm}$we have to introduce a set of auxiliary gauge fields $A \in \mathcal{G}_{<}$and $\bar{A} \in \mathcal{G}_{>}$ transforming as

$$
A \longrightarrow A^{\prime}=\alpha_{-} A \alpha_{-}^{-1}+\alpha_{-} \partial \alpha_{-}^{-1}, \quad \bar{A} \longrightarrow \bar{A}^{\prime}=\alpha_{+}^{-1} \bar{A} \alpha_{+}+\bar{\partial} \alpha_{+}^{-1} \alpha_{+} .
$$


The result is given by the gauged WZNW action (see for instance [15], [3]),

$$
\begin{aligned}
S_{G / H}(g, A, \bar{A}) & =S_{W Z N W}(g) \\
& -\frac{k}{2 \pi} \int d z^{2} \operatorname{Tr}\left(A\left(\bar{\partial} g g^{-1}-\epsilon_{+}\right)+\bar{A}\left(g^{-1} \partial g-\epsilon_{-}\right)+A g \bar{A} g^{-1}\right) .
\end{aligned}
$$

Since the action $S_{G / H}$ is $H$-invariant, we may choose $\alpha_{-}=N^{-1}$ and $\alpha_{+}=M^{-1}$. From the orthogonality of the graded subspaces, i.e. $\operatorname{Tr}\left(\mathcal{G}_{i} \mathcal{G}_{j}\right)=0, i+j \neq 0$, we find

$$
\begin{aligned}
S_{G / H}(g, A, \bar{A}) & =S_{G / H}\left(B, A^{\prime}, \bar{A}^{\prime}\right) \\
& =S_{W Z N W}(B)-\frac{k}{2 \pi} \int d z^{2} \operatorname{Tr}\left[A^{\prime} \epsilon_{+}+\bar{A}^{\prime} \epsilon_{-}+A^{\prime} B \bar{A}^{\prime} B^{-1}\right]
\end{aligned}
$$

where

$$
S_{W Z N W}=-\frac{k}{4 \pi} \int d^{2} z \operatorname{Tr}\left(g^{-1} \partial g g^{-1} \bar{\partial} g\right)-\frac{k}{24 \pi} \int_{D} \epsilon_{i j k} \operatorname{Tr}\left(g^{-1} \partial_{i} g g^{-1} \partial_{j} g g^{-1} \partial_{k} g\right),
$$

and the topological term denotes a surface integral over a ball $D$ identified as space-time.

The action (2.4) describes nonsingular Toda models among which we find the conformal and the affine abelian Toda models for $Q=\sum_{i=1}^{r} \frac{2 \lambda_{i} \cdot H}{\alpha_{i}^{2}}, \quad \epsilon_{ \pm}=\sum_{i=1}^{r} c_{ \pm i} E_{ \pm \alpha_{i}}$ and $Q=$ $h \hat{d}+\sum_{i=1}^{r} \frac{2 \lambda_{i} \cdot H}{\alpha_{i}^{2}}, \quad \hat{\epsilon}_{ \pm}=\sum_{i=1}^{r} c_{ \pm i} E_{ \pm \alpha_{i}}^{(0)}+E_{ \pm \alpha_{0}}^{( \pm 1)}$ respectively. In both cases the zero grade subgroup $\mathcal{G}_{0}=U(1)^{r}$ is abelian and it coincides with the Cartan subalgebra of $\mathcal{G}_{r}$. Performing the integration over the auxiliary fields $A$ and $\bar{A}$ in the functional integral

$$
Z_{ \pm}=\int D A D \bar{A} \exp \left(-F_{ \pm}\right)
$$

where

$$
F_{ \pm}=-\frac{k}{2 \pi} \int\left(\operatorname{Tr}\left(A-B \epsilon_{-} B^{-1}\right) B\left(\bar{A}-B^{-1} \epsilon_{+} B\right) B^{-1}\right) d^{2} z
$$

we derive the effective action for the conformal abelian Toda theories

$$
S=S_{W Z N W}(B)-\frac{k}{2 \pi} \int \operatorname{Tr}\left(\epsilon_{+} B \epsilon_{-} B^{-1}\right) d^{2} z
$$

and for the (nonconformal) affine abelian Toda IM by replacing $\epsilon_{ \pm}$from the finite algebra with the $\hat{\epsilon}_{ \pm}$belonging to the affine Kac - Moody algebra $\hat{\mathcal{G}}$. By construction the later (affine Toda) action describes an integrable perturbation of the $\mathcal{G}$-conformal abelian Toda model.

More interesting conformal (and affine) Toda models arise when the grading structure (defined by the operator Q) leads to non abelian zero grade subalgebras $\mathcal{G}_{0} \subset \mathcal{G}$. In particular, if we supress one of the fundamental weights from $Q$, the zero grade subspace acquires a nonabelian structure $s l(2) \otimes u(1)^{r a n k \mathcal{G}-1}$. Let us consider for instance $Q=h^{\prime} \hat{d}+\sum_{i \neq a}^{r} \frac{2 \lambda_{i} \cdot H}{\alpha_{i}^{2}}$, where $h^{\prime}=0$ or $h^{\prime} \neq 0$ corresponding to the conformal or affine nonabelian (NA) - Toda models respectively. The absence of $\lambda_{a}$ in $Q$ prevents the contribution of the simple root

\footnotetext{
${ }^{1}$ by $-\alpha_{0}$ we denote the highest root, $\lambda_{i}$ - the fundamental weights, $h$ - the coxeter number of $\mathcal{G}$ and $H_{i}$ are the Cartan subalgebra generators in the Cartan - Weyl basis satisfying $\operatorname{Tr}\left(H_{i} H_{j}\right)=\delta_{i j}$.
} 
step operators $E_{ \pm \alpha_{a}}^{(0)}$ in constructing $\epsilon_{ \pm}$(or in $\hat{\epsilon}_{ \pm}$), since the generators $E_{ \pm \alpha_{a}}^{(0)}$ now belong to the zero grade $\mathcal{G}_{0}$. The form of the corresponding actions is as (2.8), but with $\epsilon_{ \pm}$and $B \in \operatorname{sl}(2) \otimes u(1)^{r a n k \mathcal{G}-1}$ as described above. They are known under the name nonsingular conformal (and affine) NA - Toda models. An important feature of these models is that they manifest an additional chiral (left and right) $U(1)$ - symmetry. The algebraic origin of this fact is in the specific graded structure that allows the existence of $\mathrm{U}(1)$ - generator $\mathcal{G}_{0}^{0}=Y \cdot H \in \hat{\mathcal{G}}_{0}$ such that $\left[Y \cdot H, \epsilon_{ \pm}\right]=0$. The first term in the action (2.8) is invariant under local (chiral) $\mathcal{G}_{0}^{0}$ - transformations by construction, while the invariance of the second (potential) term is a consequence of the defining property of $Y \cdot H$ (i.e. to commute with the $\left.\epsilon_{ \pm}\right)$. The corresponding (chiral) conserved currents $J_{Y \cdot H}$ and $\bar{J}_{Y \cdot H}$ have the standard form $J_{Y \cdot H}=\operatorname{Tr}[(Y \cdot H) J]$, and $J=g^{-1} \partial g$ and $\bar{J}=-\bar{\partial} g g^{-1}$. By gauge fixing of this symmetry one obtains singular conformal (and affine ) NA - Toda models with the number of physical fields reduced by one. The elimination of the $\mathcal{G}_{0}^{0}$ field $\mathrm{R}$ in the framework of the Hamiltonian reduction consists in imposing of the nonlocal constraints $J_{Y \cdot H}=\bar{J}_{Y \cdot H}=0$. The standard method [15] of incorporating these constraints in the functional integral (2.6) is to introduce auxiliary gauge fields $A_{0}=(Y . H) a_{0}$ and $\bar{A}_{0}=(Y . H) \bar{a}_{0}$ in the action (2.8) in such a way that the constraints appear as equations of motion of the improved action. As is well known [15] the improvement consists in adding new $A_{0}, \bar{A}_{0^{-}}$dependent terms in the manner that the new action to be invariant under the following $\mathcal{G}_{0}^{0}$-transformations

$$
g \longrightarrow g^{\prime}=\alpha_{0} g \alpha_{0}^{\prime}, \quad A_{0} \longrightarrow A_{0}^{\prime}=A_{0}-\alpha_{0}^{-1} \partial \alpha_{0}, \quad \bar{A}_{0} \longrightarrow \bar{A}_{0}^{\prime}=\bar{A}_{0}-\bar{\partial} \alpha_{0}^{\prime}\left(\alpha_{0}^{\prime}\right)^{-1}
$$

There exist two inequivalent cases of gauge fixing of $\mathcal{G}_{0}^{0}=U(1)$ - symmetry, namely the axial gauging where $\alpha_{0}^{\prime}=\alpha_{0}(z, \bar{z}) \in \mathcal{G}_{0}^{0}$ and the vector gauging, where $\alpha_{0}^{\prime}=\alpha_{0}{ }^{-1}(z, \bar{z}) \in \mathcal{G}_{0}^{0}$. Finaly, the improved action with all these properties has the form [15], [7] :

$$
\begin{aligned}
S\left(B, A_{0}, \bar{A}_{0}\right) & =S\left(g_{0}^{f}, A_{0}, \bar{A}_{0}\right)=S_{W Z N W}(B) \\
& -\frac{k}{2 \pi} \int \operatorname{Tr}\left( \pm A_{0} \bar{\partial} B B^{-1}+\bar{A}_{0} B^{-1} \partial B \pm A_{0} B \bar{A}_{0} B^{-1}+A_{0} \bar{A}_{0}\right) d^{2} z \\
& -\frac{k}{2 \pi} \int d^{2} z \operatorname{Tr} \hat{\epsilon}_{+} B \hat{\epsilon}_{-} B^{-1}
\end{aligned}
$$

where the \pm signs in (2.10) correspond to axial/vector gaugings respectively. For generic affine Kac - Moody algebra $\mathcal{G}_{r}$, the zero grade element $B \in \mathcal{G}_{0}$ is parametrized as follow $\mathrm{f}$

$$
B=\exp \left(\tilde{\chi} E_{-\alpha_{a}}\right) \exp \left(R Y^{j} H_{j}+\Phi(H)+\nu \hat{c}+\eta \hat{d}\right) \exp \left(\tilde{\psi} E_{\alpha_{a}}\right)
$$

where $\Phi(H)=\sum_{j=1}^{r} \sum_{i=1}^{r-1} \varphi_{i} X^{j}{ }_{i} h_{j}, Y \cdot X_{i}=\sum_{j=1}^{r} Y^{j} X^{j}{ }_{i}=0, \quad i=1, \cdots, r-1$ and $h_{j}=\frac{2 \alpha_{j} \cdot H}{\alpha_{j}^{2}}, j=1, \cdots, r$, i.e. $X_{i}$ denotes r-dimensional vectors ortogonal to the particularly choosen vector $\mathrm{Y}$. We next define the partition function of the reduced $\mathcal{G}_{0} / \mathcal{G}_{0}^{0}=$ $\left(\operatorname{sl}(2) \otimes u(1)^{\text {rank } \mathcal{G}-1}\right) / u(1)-\mathrm{NA}$ - Toda models :

$$
Z_{\text {sing }}=\int D B D A_{0} D \bar{A}_{0} e^{-S\left(B, A_{0}, \bar{A}_{0}\right)}
$$

\footnotetext{
${ }^{2}$ All algebraic notations are as in ref.[16]. The generators $H_{i}, E_{\alpha_{a}}$, when written without upper indices correspond to $H_{i}^{(0)}, E_{\alpha_{a}}^{(0)}$ respectively. The extra generators $\hat{c}$ and $\hat{d}$ in the affine case with the properties $\left[\hat{d}, E_{\alpha_{a}}^{n}\right]=n E_{\alpha_{a}}^{n}, \quad\left[\hat{d}, H_{\alpha_{a}}^{n}\right]=n H_{\alpha_{a}}^{n}, \quad\left[\hat{c}, E_{\alpha_{a}}^{n}\right]=[\hat{c}, \hat{d}]=0$ represent the center and the derivative respectively.
} 
Integrating out the auxiliary fields $A_{0}, \bar{A}_{0}$ in (2.12) we derive the effective action $S_{\text {eff }}\left(g_{0}^{f}\right)$, where $g_{0}^{f} \in \mathcal{G}_{0} / \mathcal{G}_{0}^{0}$ is parametrized by the physical fields of the axial or vector singular conformal (or affine) NA - Toda models. The explicite form of the corresponding effective actions depends on the specific algebraic data $-\mathcal{G}_{r}, Q, \epsilon_{ \pm}$, i.e. on the algebra (finite or infinite) $\mathcal{G}_{r}$ of rank $\mathrm{r}$, on the root $\alpha_{a}$ that is missed in $\mathrm{Q}$, on the choice of $Y \cdot H=\mathcal{G}_{0}^{0}$ (fixed by $\mathrm{Q}$ and the form of $\epsilon_{ \pm}$) and finaly on the way (axial or vector) the $\mathcal{G}_{0}^{0}(=U(1)$ ) is gauge fixed. An important property common for the entire family of conformal and affine singular (axial or vector) NA - Toda models with $\mathcal{G}_{0}^{0}=U(1)$ is their global U(1) Noether symmetry. Examples of singular conformal $\mathcal{G}_{r}$ - NA - Toda models of axial type have been constructed in refs. [16], [17] and [18]. In the next two subsections we generalize the conformal constructions of refs. 16 and 18 to the case of (infinite ) affine algebras as well as for the case of vector gauging of $\mathcal{G}_{0}^{0}$. The integrable models obtained in this way represent the family of singular affine 1 NA - Toda models of axial and vector type with one global U(1) symmetry. Their main characteristic appears to be the fact [9] that for imaginary coupling they admit U(1) - charged topological solitons with the electric and magnetic charges similar to the dyonic spectrum of certain 4-dimensional Yang-Mills-Higgs theory[9]. This is the reason to call them dyonic integrable models. As we shall demonstrate in Sect.3 below the fact that the axial and vector IMs are obtained from the unique nonsingular IM with local U(1) symmetry by two different gauge fixings of this symmetry, gives rise to an interesting phenomena : pairs of integrable models related by the off-critical T-duality transformation.

\subsection{Axial Gauging}

Taking into account the fact that the action (2.10) is invariant under $\mathrm{U}(1)$ transformations (2.9) we can gauge away the nonlocal field $R$ by choosing $\alpha_{0}^{\prime}=\alpha_{0}=e^{-\frac{1}{2} Y \cdot H R}$, that corresponds to axial gauge fixing. Then the gauge fixed element $\mathrm{B}$ (i.e. the factor group element $\left.g_{0}^{f} \in \mathcal{G}_{0} / \mathcal{G}_{0}^{0}\right)$ becomes

$$
g_{0}^{f}=\exp \left(\chi E_{-\alpha_{a}}\right) \exp (\Phi(H)+\nu \hat{c}+\eta \hat{d}) \exp \left(\psi E_{\alpha_{a}}\right)
$$

where $\chi=\tilde{\chi} e^{\frac{1}{2} Y \cdot \alpha_{a} R}$ and $\psi=\tilde{\psi} e^{\frac{1}{2} Y \cdot \alpha_{a} R}$. With this parametrization the $\operatorname{second}\left(A_{0}, \bar{A}_{0}\right)-$ dependent term in the action (2.10) takes the form :

$$
\begin{aligned}
F_{0} & =-\frac{k}{2 \pi} \int \operatorname{Tr}\left(A_{0} \bar{\partial} g_{0}^{f}\left(g_{0}^{f}\right)^{-1}+\bar{A}_{0}\left(g_{0}^{f}\right)^{-1} \partial g_{0}^{f}+A_{0} g_{0}^{f} \bar{A}_{0}\left(g_{0}^{f}\right)^{-1}+A_{0} \bar{A}_{0}\right) d^{2} z \\
& =-\frac{k}{2 \pi} \int\left(a_{0} \bar{a}_{0} 2 Y^{2} \Delta_{a}-\left(\frac{2 \alpha_{a} \cdot Y}{\alpha_{a}^{2}}\right)\left(\bar{a}_{0} \psi \partial \chi+a_{0} \chi \bar{\partial} \psi\right) e^{\Phi\left(\alpha_{a}\right)}\right) d^{2} z
\end{aligned}
$$

where $\Delta_{a}=1+\frac{\left(Y \cdot \alpha_{a}\right)^{2}}{\alpha_{a}^{2} Y^{2}} \psi \chi e^{\Phi\left(\alpha_{a}\right)}$ and $\left[\Phi(H), E_{\alpha_{a}}\right]=\Phi\left(\alpha_{a}\right) E_{\alpha_{a}}$. As we have mentioned the effective action is obtained by integrating over the auxiliary fields $A_{0}$ and $\bar{A}_{0}$ in the functional

\footnotetext{
${ }^{3}$ remember that in the case $\mathcal{G}_{0}$ is abelian an invariant subalgebra $\mathcal{G}_{0}^{0}$ does not exist and the corresponding affine abelian Toda models do not have any Noether symmetries.

${ }^{4}$ and conformal affine NA- Toda models when the generators $\hat{c}$ and $\hat{d}$ are included in the zero grade subalgebra
} 
integral (2.12)

$$
Z_{\text {sing }}=\int D g_{0}^{f} D A_{0} D \bar{A}_{0} \exp \left(F_{0}\right) \sim \int D g_{0}^{f} e^{-S_{0}-S_{W Z N W}\left(g_{0}^{f}\right)-S_{\text {int }}}
$$

where $S_{0}=-\frac{k}{2 \pi}\left(\frac{2 Y \cdot \alpha_{a}}{\alpha_{a}^{2}}\right)^{2} \int d^{2} z \frac{\psi \chi \bar{\partial} \psi \partial \chi}{2 Y^{2} \Delta_{a}} e^{2 \Phi\left(\alpha_{a}\right)}$ and $S_{\text {int }}=\frac{k}{2 \pi} \int d^{2} z \operatorname{Tr}\left[\hat{\epsilon}_{+} g_{0}^{f} \hat{\epsilon}_{-}\left(g_{0}^{f}\right)^{-1}\right]$. The total effective action (2.10) for the axial IM is therefore given as

$$
\begin{aligned}
S_{e f f} & =-\frac{k}{4 \pi} \int d^{2} z\left(\operatorname{Tr}(\partial \Phi(H) \bar{\partial} \Phi(H))+\frac{4 \bar{\partial} \psi \partial \chi e^{\Phi\left(\alpha_{a}\right)}}{\alpha_{a}^{2} \Delta_{a}}\right. \\
& \left.+\partial \eta \bar{\partial} \nu+\partial \nu \bar{\partial} \eta-2 \operatorname{Tr}\left(\hat{\epsilon_{+}} g_{0}^{f} \hat{\epsilon_{-}}\left(g_{0}^{f}\right)^{-1}\right)\right)
\end{aligned}
$$

Note that the second term in (2.16) contains both symmetric and antisymmetric parts:

$$
\frac{e^{\Phi\left(\alpha_{a}\right)}}{\Delta_{a}} \bar{\partial} \psi \partial \chi=\frac{e^{\Phi\left(\alpha_{a}\right)}}{\Delta_{a}}\left(g^{\mu \nu} \partial_{\mu} \psi \partial_{\nu} \chi+\epsilon_{\mu \nu} \partial_{\mu} \psi \partial_{\nu} \chi\right)
$$

where $g_{\mu \nu}$ is the $2-\mathrm{D}$ metric of signature $g_{\mu \nu}=\operatorname{diag}(1,-1), \partial=\partial_{0}+\partial_{1} \bar{\partial}=\partial_{0}-\partial_{1}$. For $r=1\left(\mathcal{G} \equiv A_{1}^{(1)}, \Phi\left(\alpha_{1}\right)\right.$ is zero) the antisymmetric term is a total derivative:

$$
\epsilon_{\mu \nu} \frac{\partial_{\mu} \psi \partial_{\nu} \chi}{1+\psi \chi}=\frac{1}{2} \epsilon_{\mu \nu} \partial_{\mu}\left(\ln \{1+\psi \chi\} \partial_{\nu} \ln \frac{\chi}{\psi}\right),
$$

and it can be neglected. This $A_{1}$-NA-Toda model (in the conformal case), is known to describe the 2-D black hole solution for (2-D) string theory [19]. The G-NA conformal Toda models can be used in the description of specific $(\mathrm{r}+1)$-dimensional black string theories [20], [7] with (r-1)-flat and 2-nonflat directions $\left(g^{\mu \nu} G_{a b}(X) \partial_{\mu} X^{a} \partial_{\nu} X^{b}, X^{a}=\left(\psi, \chi, \varphi_{i}\right)\right)$, containing axions $\left(\epsilon_{\mu \nu} B_{a b}(X) \partial_{\mu} X^{a} \partial_{\nu} X^{b}\right)$ and tachyons $\left(\exp \left\{-k_{i j} \varphi_{j}\right\}\right)$, as well. One particular example of dyonic axial IM based on $A_{r}^{(1)}$ for $a=1$, i.e. $Y \cdot H=\lambda_{1} \cdot H$ will be discussed in Sect.3.

\subsection{Vector Gauging}

The vector gauging is implemented by choosing $\alpha_{0}^{\prime}=\alpha_{0}^{-1}=e^{\frac{1}{2} Y \cdot H R^{\prime}}$, where $\bar{\chi}=-e^{\frac{1}{2} Y \cdot \alpha_{a} R^{\prime}} \tilde{\chi}=$ $e^{-\frac{1}{2} Y \cdot \alpha_{a} R^{\prime}} \tilde{\psi}$, i.e. $R^{\prime}=\frac{1}{Y \cdot \alpha_{a}} \ln \left(\frac{-\tilde{\psi}}{\tilde{\chi}}\right)$. Then the factor group element can be parametrized as

$$
g_{0}^{f}=\exp \left(-\bar{\chi} E_{-\alpha_{a}}\right) \exp (\Phi(H)+\nu \hat{c}+\eta \hat{d}) \exp \left(\bar{\chi} E_{\alpha_{a}}\right)
$$

where $\Phi(H)=Y \cdot H R+\sum_{j=1}^{r-1} \varphi_{j} X_{j}^{i} h_{i}$. The second term of the action (2.10) then takes the form :

$$
\begin{aligned}
F_{0} & =-\frac{k}{2 \pi} \int\left(-\frac{2}{\alpha_{a}^{2}}\left(Y \cdot \alpha_{a}\right)^{2} a_{0} \bar{a}_{0} \bar{\chi}^{2} e^{\Phi\left(\alpha_{a}\right)}-\bar{a}_{0}\left(Y^{2} \partial R+\frac{2 Y \cdot \alpha_{a}}{\alpha_{a}^{2}} \bar{\chi} \partial \bar{\chi} e^{\Phi\left(\alpha_{a}\right)}\right)\right. \\
& +a_{0}\left(Y^{2} \bar{\partial} R+\frac{2 Y \cdot \alpha_{a}}{\alpha_{a}^{2}} \bar{\chi} \bar{\partial} \bar{\chi} e^{\Phi\left(\alpha_{a}\right)}\right) d^{2} z
\end{aligned}
$$


Integrating over $a_{0}$ and $\bar{a}_{0}$ in (2.12) we derive the total effective action (2.10) for the vector gauged IM :

$$
\begin{aligned}
S_{e f f} & =-\frac{k}{4 \pi} \int\left(\sum_{i, j=1}^{r-1} \operatorname{Tr}\left(X_{i} \cdot h X_{j} \cdot h\right) \partial \varphi_{i} \bar{\partial} \varphi_{j}+Y^{2} \partial R \bar{\partial} R+\partial \eta \bar{\partial} \nu+\partial \nu \bar{\partial} \eta\right. \\
& \left.+2 \frac{2}{\alpha_{a}^{2}} \rho^{2}\left(\alpha_{a}\right) \frac{\partial R \bar{\partial} R}{\bar{\chi}^{2}} e^{-\Phi\left(\alpha_{a}\right)}+2 \frac{2}{\alpha_{a}^{2}} \rho\left(\alpha_{a}\right)(\partial R \bar{\partial} \ln \bar{\chi}+\bar{\partial} R \partial \ln \bar{\chi})-2 \operatorname{Tr}\left(\hat{\epsilon_{+}} g_{0}^{f} \hat{\epsilon_{-}}\left(g_{0}^{f}\right)^{-1}\right)\right) d^{2} z
\end{aligned}
$$

where $\rho\left(\alpha_{a}\right)=\frac{Y^{2} \alpha_{a}^{2}}{2\left(Y \cdot \alpha_{a}\right)}$. Defining the new variables

$$
E=e^{\gamma R}, \quad F=E^{-1}\left(1-\delta \bar{\chi}^{2} \exp \Phi\left(\alpha_{a}\right)\right)
$$

the action (2.19) becomes

$$
\begin{aligned}
S_{e f f} & =-\frac{k}{2 \pi} \int\left(\frac{1}{2} \sum_{i, j=1}^{r-1} \operatorname{Tr}\left(X_{i} \cdot h X_{j} \cdot h\right) \partial \varphi_{i} \bar{\partial} \varphi_{j}+\frac{1}{2 \gamma^{2}} \partial \ln E \bar{\partial} \ln E\left(Y^{2}+4 \gamma^{2} \Gamma\right)\right. \\
& -\Gamma\left(\partial \ln E \bar{\partial} \Phi\left(\alpha_{a}\right)+\bar{\partial} \ln E \partial \Phi\left(\alpha_{a}\right)\right)+\frac{1}{2} \partial \eta \bar{\partial} \nu+\frac{1}{2} \partial \nu \bar{\partial} \eta-\Gamma \frac{(\partial E \bar{\partial} F+\bar{\partial} E \partial F)}{1-E F} \\
& \left.-\operatorname{Tr}\left(\hat{\epsilon_{+}} g_{0}^{f} \hat{\epsilon_{-}}\left(g_{0}^{f}\right)^{-1}\right)\right) d^{2} z
\end{aligned}
$$

where $2 \gamma \Gamma=\frac{2}{\alpha_{a}^{2}} \rho\left(\alpha_{a}\right)$ and $2 \delta \Gamma=\frac{2}{\alpha_{a}^{2}}$ are chosen in order to eliminate the variable $\bar{\chi}$. Notice that the $E, F$-term in the action (2.21) is symmetric and contrary to the axial model the above action is CPT - invariant. The vector gauging, therefore provides a construction of torsionless actions T-dual to its axionic counterpart as we shall demonstrate in Sect. 3. This fact raises the question whether exist T-selfdual torsionless actions, i.e.when the axial and vector gauging leads to same action. It is worthwhile to mention that the dyonic integrable models of vector type (2.21) represent integrable perturbations of the conformal $\sigma$ - models studied in [7] in the context of the string backgrounds of black hole type .

\subsubsection{Example1. Torsionless $B_{r}^{(1)}$ model}

We consider the particular case based on $B_{r}^{(1)}$ by taking $Y=\frac{2 \lambda_{r}}{\alpha_{r}^{2}}-\frac{2 \lambda_{r-1}}{\alpha_{r-1}^{2}}=e_{r}, a=r, \rho\left(\alpha_{r}\right)=$ $\frac{1}{2}$, where

$$
\lambda_{r}=\frac{1}{2}\left(e_{1}+e_{2}+\cdots+e_{r}\right), \quad \lambda_{r-1}=\left(e_{1}+e_{2}+\cdots+e_{r-1}\right)
$$

and

$$
\epsilon_{ \pm}=\sum_{i=1}^{r-2} E_{ \pm \alpha_{i}}^{(0)}+E_{ \pm\left(\alpha_{r-1}+\alpha_{r}\right)}^{(0)}+E_{\mp\left(\alpha_{1}+2\left(\alpha_{2}+\cdots \alpha_{r-1}+\alpha_{r}\right)\right)}^{( \pm 1)}
$$


Parametrizing the Cartan part of $g_{0}^{f}$ as $\Phi(H)=\sum_{i=1}^{r-1} \varphi_{i} e_{i} \cdot H+2\left(e_{r} \cdot H\right) \ln E$, we find

$$
\begin{aligned}
S_{\text {eff }} & =-\frac{k}{2 \pi} \int\left(\frac{1}{2} \sum_{i, j=1}^{r-1} \partial \varphi_{i} \bar{\partial} \varphi_{i}+\frac{1}{2} \partial \eta \bar{\partial} \nu+\frac{1}{2} \partial \nu \bar{\partial} \eta-\frac{(\partial E \bar{\partial} F+\bar{\partial} E \partial F)}{1-E F}\right. \\
& \left.-\operatorname{Tr}\left(\hat{\epsilon_{+}} g_{0}^{f} \hat{\epsilon_{-}}\left(g_{0}^{f}\right)^{-1}\right)\right) d^{2} z
\end{aligned}
$$

where we have chosen $\Gamma=1, \gamma=\frac{1}{2}, \delta=1$. When the pair of fields $\eta$ and $\nu$ are taken to be equal to zero the corresponding conformal affine $B_{r}^{(1)} \mathrm{NA}$ - Toda model takes the form of the nonconformal affine $B_{r}^{(1)} \mathrm{NA}$ - Toda model.

\subsubsection{Example2. $A_{r}^{(1)}$ vector model}

In order to find the explicit form of the singular affine NA - Toda IM based on the affine algebra $A_{r}^{(1)}$ we take $Y=\lambda_{1}$ and $\epsilon_{ \pm}=\sum_{i=2}^{r} E_{ \pm \alpha_{i}}^{(0)}+E_{\mp\left(\alpha_{2}+\cdots \alpha_{r}\right)}^{( \pm 1)}$. We will get simpler result if we parametrize $\Phi(H)=\sum_{i=1}^{r} \phi_{i} h_{i}$ :

$$
\begin{aligned}
S_{e f f} & =-\frac{k}{4 \pi} \int\left(\sum_{i, j=1}^{r} \eta_{i j} \partial \phi_{i} \bar{\partial} \phi_{j}+2 \frac{\partial \phi_{1} \bar{\partial} \phi_{1}}{\bar{\chi}^{2} e^{2 \phi_{1}-\phi_{2}}}\right. \\
& \left.+2\left(\partial \phi_{1} \bar{\partial} \ln \bar{\chi}+\bar{\partial} \phi_{1} \partial \ln \bar{\chi}\right)-2 \operatorname{Tr}\left(\hat{\epsilon_{+}} g_{0}^{f} \hat{\epsilon_{-}}\left(g_{0}^{f}\right)^{-1}\right)\right) d^{2} z
\end{aligned}
$$

With the following change of the field variables

$$
E=e^{\phi_{1}}, \quad F=E^{-1}\left(1-\bar{\chi}^{2} e^{2 \phi_{1}-\phi_{2}}\right)
$$

we find

$$
S_{e f f}=-\frac{k}{2 \pi} \int\left(\frac{1}{2} \sum_{i, j=2}^{r} \eta_{i j} \partial \phi_{i} \bar{\partial} \phi_{j}-\frac{1}{2} \frac{(\partial E \bar{\partial} F+\bar{\partial} E \partial F)}{1-E F}-\operatorname{Tr}\left(\hat{\epsilon_{+}} g_{0}^{f} \hat{\epsilon_{-}}\left(g_{0}^{f}\right)^{-1}\right)\right) d^{2} z
$$

Introducing new fields $c_{i}=e^{\phi_{i+2}-\phi_{i+1}}, \quad i=1, \cdots r-1$ we rewrite the action (2.26) in the form

$$
\begin{aligned}
& S_{\text {eff }}=-\frac{k}{2 \pi} \int\left(\frac{1}{2} \sum_{i=1}^{r-1}\left(\partial \ln c_{i} \bar{\partial} \ln c_{1} c_{i+1} \cdots c_{r-1}+\bar{\partial} \ln c_{i} \partial \ln c_{1} c_{i+1} \cdots c_{r-1}\right)\right. \\
& \left.-\frac{1}{2} \frac{(\partial E \bar{\partial} F+\bar{\partial} E \partial F)}{1-E F}-\operatorname{Tr}\left(\hat{\epsilon_{+}} g_{0}^{f} \hat{\epsilon_{-}}\left(g_{0}^{f}\right)^{-1}\right)\right) d^{2} z
\end{aligned}
$$

These are the vector IM's studied in ref. [9] .

\section{Off-Critical T-Duality}

T-duality 10 is known to be an important property of the string theory. It acts as canonical transformation [23], 110] in the string phase space $\mathcal{P}=\left\{X^{M}(\sigma), \Pi_{M}(\sigma)=g_{M N} \dot{X}^{N}+\right.$ 
$\left.b_{M}^{N} X_{N}^{\prime} ; b, c\right\}$ mapping the original conformal $\sigma$-model [:

$$
S_{\sigma}^{\text {conf }}=\frac{1}{4 \pi \alpha^{\prime}} \int d^{2} z\left(\left(g_{M N}(X) \eta^{\mu \nu}+b_{M N}(X) \epsilon^{\mu \nu}\right) \partial_{\mu} X^{M} \partial_{\nu} X^{N}+\frac{\alpha^{\prime}}{2} R^{(2)} \varphi(X)\right)
$$

$\left(\mu, \nu=0,1 ; M, N=1,2, \cdots D\right.$ and $R^{(2)}$ is the worldsheet curvature ) to its T-dual model $\tilde{S}_{\sigma}^{\text {conf }}\left(G_{M N}(\tilde{X}), B_{M N}(\tilde{X}), \phi(\tilde{X})\right)$. Curved string backgrounds with d-isometric directions provide an example of an abelian T-duality transformation 24, 10]:

$$
\begin{aligned}
E_{\alpha \beta} & =\left(e^{-1}\right)_{\alpha \beta}, \quad E_{m n}=e_{m n}-e_{m \alpha}\left(e^{-1}\right)^{\alpha \beta} e_{\beta n} \\
E_{\alpha m} & =\left(e^{-1}\right)_{\alpha}^{\beta} e_{\beta m}, \quad E_{m \alpha}=-e_{m \beta}\left(e^{-1}\right)_{\alpha}^{\beta} \\
\phi & =\varphi-\ln \left[\operatorname{det} E_{\alpha \beta}\right], \quad \alpha, \beta=1,2, \cdots d, \quad m, n=d+1, \cdots D
\end{aligned}
$$

where $E_{M N}=G_{M N}+B_{M N}$. The canonical transformation $\left(\Pi_{X}, X\right) \rightarrow\left(\Pi_{\tilde{X}}, \tilde{X}\right)$ that generates the background maps (3.2) has the following simple form [23]:

$$
\Pi_{\tilde{X}_{\alpha}}=-X_{\alpha}^{\prime}, \quad \Pi_{X_{\alpha}}=-\tilde{X}_{\alpha}^{\prime}
$$

and all the $\Pi_{X_{m}}$ and $X_{m}$ remain unchanged. By construction both $\sigma$-models $S_{\sigma}^{\text {conf }}(e, \varphi)$ and $\tilde{S}_{\sigma}^{\text {conf }}(E, \phi)$ have coinciding energy spectrum and partition functions. The corresponding Lagrangeans are related by the generating function $\mathcal{F}$ :

$$
\begin{aligned}
\mathcal{L}(e, \varphi) & =\mathcal{L}(E, \phi)+\frac{d \mathcal{F}}{d t}, \quad \mathcal{F}=\frac{1}{8 \pi \alpha^{\prime}} \int d x\left(X \cdot \tilde{X}^{\prime}-X^{\prime} \cdot \tilde{X}\right), \\
\frac{\delta \mathcal{F}}{\delta X^{\alpha}} & =\Pi_{X_{\alpha}}, \quad \frac{\delta \mathcal{F}}{\delta \tilde{X}^{\alpha}}=-\Pi_{\tilde{X}_{\alpha}}
\end{aligned}
$$

An important feature of the abelian T-duality (3.2) and (3.3) is that it maps the $U(1)^{d}$ Noether charges $Q^{\alpha}=\int_{-\infty}^{\infty} J_{0}^{\alpha} d x$ of $S_{\sigma}^{\text {conf }}$ into the topological charges $\tilde{Q}_{t o p}^{\alpha}=\int_{-\infty}^{\infty} d x \partial_{x} \tilde{X}^{\alpha}$ of the dual model $\tilde{S}_{\sigma}^{\text {conf }}$ :

$$
\begin{aligned}
J_{\mu}^{\alpha} & =\frac{1}{2} e^{\alpha \beta}\left(X_{n}\right) \partial_{\mu} X_{\beta}+\frac{1}{2} e^{\alpha m}\left(X_{n}\right) \partial_{\mu} X_{m} \equiv \frac{1}{2} \epsilon_{\mu \nu} \partial^{\nu} \tilde{X}^{\alpha} \\
\tilde{J}_{\mu}^{\alpha} & =\frac{1}{2} E^{\alpha \beta}\left(\tilde{X}_{n}\right) \partial_{\mu} \tilde{X}_{\beta}+\frac{1}{2} E^{\alpha m}\left(\tilde{X}_{n}\right) \partial_{\mu} \tilde{X}_{m} \equiv \frac{1}{2} \epsilon_{\mu \nu} \partial^{\nu} X^{\alpha}
\end{aligned}
$$

i.e. $\left(Q^{\alpha}, Q_{t o p}^{\alpha}\right) \rightarrow\left(\tilde{Q}_{t o p}^{\alpha}, \tilde{Q}^{\alpha}\right)$. It is well known [10], [7] that the main part of the conformal $\sigma$-models representing relevant string backgrounds can be derived from the axial or vector gauged $G / H$-WZNW models. All the models constructed in Sect.2 with vanishing potential term $V=\frac{m^{2} k}{2 \pi} \operatorname{Tr}\left(\epsilon_{+} B \epsilon_{-} B^{-1}\right)$ (i.e. $\left.m=0\right)$ are of this type. They have $d=r$ isometric directions, i.e. $e_{m n}$ do not depend on $\varphi_{i},(i=2, \cdots, r)$ and $\theta=\frac{1}{2} \ln \frac{\psi}{\chi}$. The T-duality group in this case is known to be $O(r, r \mid Z)$ (see for example [10]). Adding the potential $V$ with $\epsilon_{ \pm}=\sum_{i \neq a} E_{ \pm \alpha_{i}}^{(0)}$ (see eqs. (2.16) and (2.21)) specific for the conformal NA -Toda theories

\footnotetext{
${ }^{5}$ under certain symmetry restrictions on the geometrical data: $e_{M N}(X)=g_{M N}(X)+b_{M N}(X)$ and $\varphi(X)$. In the case of abelian T-duality $e_{M N}(X)$ and are $\varphi(X)$ independent of $d \leq D$ of $X_{\alpha},(\alpha=1,2, \cdots d \leq D)$ called isometric target-space coordinates.
} 
with one global U(1) symmetry, we are decreasing the number of the isometric coordinates from $d=r$ to $d_{0}=1$. Taking $\epsilon_{ \pm}=\sum_{i \neq a, b} E_{ \pm \alpha_{i}}^{(0)}$ one can construct NA - Toda theories with $d_{0}=2$, etc.

The problem we are addressing in this section is about the T-duality between $d_{0}=1$ axial and vector integrable (nonconformal) models of Sect.2.1 and Sect.2.2 with potential terms constructed by taking

$$
\begin{aligned}
\hat{\epsilon}_{ \pm} & =\sum_{i=2}^{r} E_{ \pm \alpha_{i}}^{(0)}+E_{\mp\left(\alpha_{2}+\cdots+\alpha_{r}\right)}^{( \pm 1)} \\
V_{a} & =\frac{m^{2} k}{2 \pi}\left(\sum_{i=2}^{r} e^{\varphi_{i-2}+\varphi_{i}-2 \varphi_{i-1}}+e^{\varphi_{2}+\varphi_{r}}\left(1+\psi \chi e^{-\varphi_{2}}\right)\right), \quad \varphi_{0}=\varphi_{r+1}=0 \\
V_{v e c} & =\frac{m^{2} k}{2 \pi}\left(\sum_{i=3}^{r} e^{\phi_{i-1}+\phi_{i+1}-2 \phi_{i}}+E e^{-2 \phi_{2}+\phi_{3}}+F e^{\phi_{2}+\phi_{r}}\right),
\end{aligned}
$$

for the $A_{r}^{(1)}$ model of Example 2. For the $B_{r}^{(1)}$ model (with $\eta=0$ ) of Example 1 (see also Sect.4.2) we have

$$
\hat{\epsilon}_{ \pm}=\sum_{i=1}^{r-2} E_{ \pm \alpha_{i}}^{(0)}+E_{ \pm\left(\alpha_{r-1}+\alpha_{r}\right)}^{(0)}+E_{ \pm \alpha_{0}}^{( \pm 1)}, \quad \alpha_{0}+\alpha_{1}+2\left(\alpha_{2}+\cdots \alpha_{r-1}+\alpha_{r}\right)=0
$$

with potential given by eq. (4.7) for the axial model. Its vector counterpart is given by

$$
V_{v e c}^{B_{r}^{1}}=\sum_{i=1}^{r-2}\left|c_{i}\right|^{2} e^{-\varphi_{i}+\varphi_{i+1}}+2\left|c_{r-1}\right|^{2}(2 E F-1) e^{-\varphi_{r-1}}+\left|c_{r}\right|^{2} e^{\varphi_{1}+\varphi_{2}}
$$

In both cases the axial IM's isometric coordinate is $X=\theta=\frac{1}{2} \ln \frac{\chi}{\psi}\left(\tilde{u}^{2}=\frac{\psi}{\chi}\right)$. For the corresponding vector IM's we choose $\tilde{X}_{B_{r}^{(1)}}=R_{B}=2 \ln E$ and $\tilde{X}_{A_{r}^{(1)}}=R_{A}=\frac{r+1}{r} \ln E$ as isometric coordinates. It is important to mention that in the case of $A_{r}^{(1)}$ vector model the canonical transformation (3.3) with $d=1$ has to be accompanied by the following point transformation:

$$
\phi_{j}=\phi_{j}^{\prime}-\frac{r-j+1}{2 r} R_{A}, \quad j=2, \cdots r
$$

Then performing $d_{0}=1$ T-duality transformation (3.2) (together with the $A_{r}^{(1)}$ fields transformation (3.8)) we realize that $\mathcal{L}_{\text {vec }}$ and $\mathcal{L}_{a}$ given by eqs. (2.21) and (2.16), with potentials (3.6) and (4.7), are related by eq.(3.4) with

$$
\frac{d \mathcal{F}}{d t}=2 \Gamma(\partial \ln \tilde{u} \bar{\partial} \ln E-\bar{\partial} \ln \tilde{u} \partial \ln E)
$$

Notice that the $B_{r}^{(1)}$ vector and axial Lagrangeans have the same form, i.e. they are Tselfdual.

An alternative way to perform the T-duality transformation between $d_{0}=1$ axial and vector IM's in consideration consists in making the following nonlocal change of the field variables: 
a) $A_{r}^{(1)}$ case

$$
E=e^{\frac{r}{r+1} R_{A}}, \quad F=e^{-\frac{r}{r+1} R_{A}}\left(1+\psi \chi e^{-\varphi_{2}}\right), \quad \phi_{j}=\varphi_{j}+\frac{r-j+1}{r} R_{A}, \quad j=2, \cdots r
$$

b) $B_{r}^{(1)}$ case

$$
E=e^{\frac{1}{2} R_{B}}, \quad F=e^{-\frac{1}{2} R_{B}}(1+\psi \chi), \quad \phi_{j}=\varphi_{j}
$$

instead of the canonical transformation(3.3) ( resulting in (3.2)). Eqs. (3.9) and (3.10) in fact represent the integrated form of (3.3). Their derivation (see Sect.5 of ref. [9]) is based on the comparison of the $g_{0}$ (or $B$ ) group elements written in axial and vector parametrizations (2.13) and (2.17), i.e. imposing $g_{0}^{v e c}=g_{0}^{a x}$. An important ingredient of this calculation are the relations (3.5) between the $U(1)$ currents and the topological currents $\epsilon^{\mu \nu} \partial_{\nu} R$ (and $\left.\epsilon^{\mu \nu} \partial_{\nu} \theta\right)$. Note that $R=2 \ln E$ is a nonlocal (nonphysical) field in the axial model, but it appears to be physical in the vector model. In the case of the $B_{r}^{(1)}$ model (2.23) the $U(1) \leftrightarrow$ topological-currents relations (3.5) take the following explicit form:

$$
\begin{aligned}
\partial \ln \tilde{u} & =\frac{v}{v-1} \partial \ln E-\frac{1}{2} \frac{\partial v}{v-1} \\
\bar{\partial} \ln \tilde{u} & =-\frac{v}{v-1} \bar{\partial} \ln E+\frac{1}{2} \frac{\bar{\partial} v}{v-1}
\end{aligned}
$$

where $v=E F$ and $\tilde{u}^{2}=\frac{\chi}{\psi}$.

Although the T-duality between the vector and axial integrable models is quite similar to the conformal "free" case (i.e. $V=0)$ 的 the off-critical T-duality addresses few new problems specific for the integrable models. In the case of imaginary coupling constant $\beta^{2}=-\frac{2 \pi}{k}$, i.e. $\beta \rightarrow i \beta_{0}$ and $\varphi_{k} \rightarrow i \beta_{0} \varphi_{k}, \psi \rightarrow i \beta_{0} \psi$, etc. one expects that both axial and vector IM's possess soliton solutions. One might wonder what is the relation between the solitons (and breathers ) of the T-dual integrable models, whether their soliton spectra coincides (modulo the interchanges $\left.Q \rightarrow \tilde{Q}_{t o p}, \quad \tilde{Q} \rightarrow Q_{t o p}\right)$ and finally about the $O(1,1 \mid Z)$ symmetry of the solitons energies and massess. Partial answer of all these questions is presented in our recent work [9].

\section{No Torsion Theorem}

It becomes clear from our discussion in Sect.3 that the abelian T-duality between the axial and the vector dyonic IM's allows to single out two classes of models : (a) T-selfdual torsionless IM's and (b) pairs of T-duals axial and vector IM's. One may wonder what is the Lie algebraic condition defining the models of class (a). In answering this question we first establish the T-selfduality condition for the conformal $d_{0}=1$ (i.e $\mathcal{G}_{0}^{0}=U(1)$ ) singular NA Toda models. We next generalize the conformal no torsion (T-selfduality) theorem to the case of U(1) symmetric affine NA Toda IM's of sects. 4.2 and 4.3.

\footnotetext{
${ }^{6}$ the only new feature is that one should take care about the specific "point" transformations involving the potential $V$ and that the isometric coordinates are reduced from $d=r$ to $d_{0}=1$.
} 


\subsection{Conformal T-selfdual NA Toda models}

T-selfduality requires that axial and vector models have coinciding Hamiltonians. Since the vector models are by construction torsionless the question to be addressed is about the algebraic condition under which the axial gauging generate torsionless models as well.

Consider a finite dimensional Lie algebra $\mathcal{G}$ with grading operator given by $Q_{a}=\sum_{i \neq a}^{r} \frac{2}{\alpha_{i}^{2}} \lambda_{i}$. $H$ and take the most general constant generators of grade \pm 1 , i. e.,

$$
\epsilon_{ \pm}=\sum_{i \neq a}^{r} c_{ \pm i} E_{ \pm \alpha_{i}}+b_{ \pm} E_{ \pm\left(\alpha_{a}+\alpha_{a+1}\right)}+d_{ \pm} E_{ \pm\left(\alpha_{a}+\alpha_{a-1}\right)} .
$$

It is clear that if $c_{ \pm i}, b_{ \pm}, d_{ \pm} \neq 0$, there shall be no $\mathcal{G}_{0}^{0}$ commuting with $\epsilon_{ \pm}$, since that requires an orthogonal direction to all roots appearing in $\epsilon_{ \pm}$. These are the generalized non-singular NA-Toda models of ref. [3], [6]. The NA-Toda models of singular metric $G_{i j}(X)$ correspond to the cases when $\mathcal{G}_{0}^{0}=U(1)$ ( or $U(1)^{r}$ in general ) and $J_{Y \cdot H}=0=\bar{J}_{Y \cdot H}$ imposed as a subsidiary constraint \. Depending on the choice of the constants $c_{ \pm i}, b_{ \pm}$and $d_{ \pm}$we distinguish four families of singular conformal NA-Toda models:

$$
\begin{aligned}
& \text { (i) } b_{ \pm}=d_{ \pm}=0, \mathcal{G}_{0}^{0}=\frac{2}{\alpha_{a}^{2}} \lambda_{a} \cdot H ; \\
& \text { (ii) } c_{ \pm(a-1)}=c_{ \pm(a+1)}=0, \mathcal{G}_{0}^{0}=\frac{2}{\alpha_{a}^{2}} \lambda_{a} \cdot H-\frac{2}{\alpha_{a-1}^{2}} \lambda_{a-1} \cdot H-\frac{2}{\alpha_{a+1}^{2}} \lambda_{a+1} \cdot H ; \\
& \text { (iii) } c_{ \pm(a+1)}=d_{ \pm}=0, \mathcal{G}_{0}^{0}=\frac{2}{\alpha_{a}^{2}} \lambda_{a} \cdot H-\frac{2}{\alpha_{a+1}^{2}} \lambda_{a+1} \cdot H ; \\
& \text { (iv) } b_{ \pm}=c_{ \pm(a-1)}=0, \mathcal{G}_{0}^{0}=\frac{2}{\alpha_{a}^{2}} \lambda_{a} \cdot H-\frac{2}{\alpha_{a-1}^{2}} \lambda_{a-1} \cdot H .
\end{aligned}
$$

Of course, if $c_{ \pm j}=0, j \neq a, a \pm 1$, we find $\mathcal{G}_{0}^{0}=\lambda_{j} \cdot H$. However, since $\left[\lambda_{j} \cdot H, E_{ \pm \alpha_{a}}\right]=0$, there will be no singular metric present and this case shall be neglected. Cases (i) and (ii) are equivalent, since they are related by the Weyl reflection $\sigma_{\alpha_{a}}\left(\alpha_{a \pm 1}\right)=\alpha_{a}+\alpha_{a \pm 1}$ and the corresponding fields are related by non-linear change of the variables. This case has already been discussed in refs. [17] and [18], and shown to present always the antisymmetric term, originated by the presence of $e^{k_{a i} \varphi_{i}}$ in $\Delta_{a}$ and in the kinetic term as well. Since we are removing all dependence in $\mathcal{G}_{0}^{0}$, when parameterizing $g_{0}^{f}$, cases (iii) and (iv) may be studied together with

$$
g_{0}^{f}=\exp \left(\chi E_{-\alpha_{a}}\right) \exp (\Phi(H)) \exp \left(\psi E_{\alpha_{a}}\right)
$$

where $\Phi(H)=\sum_{i=1}^{a-2} \varphi_{i} h_{i}+\varphi_{-}\left(\chi_{-} \cdot H\right)+\varphi_{+}\left(\chi_{+} \cdot H\right)+\sum_{i=a+2}^{r} \varphi_{i} h_{i}$,

$$
\chi_{-}^{(i i i)}=\alpha_{a-1}+\alpha_{a}, \quad \chi_{+}^{(i i i)}=\alpha_{a+1}, \quad, \quad \chi_{-}^{(i v)}=\alpha_{a-1}, \quad \chi_{+}^{(i v)}=\alpha_{a}+\alpha_{a+1}
$$

for cases $(i i i)$ and $(i v)$ respectively, and $\mathcal{G}_{0}^{0}=Y \cdot H$, such that $\operatorname{Tr}\left(\chi_{ \pm} \cdot H \mathcal{G}_{0}^{0}\right)=0$. Such parametrization of $g_{0}^{f}$ yields

$$
\Phi\left(\alpha_{a}\right)=\sum_{i=1}^{a-2} k_{a i} \varphi_{i}+\left(\alpha_{a} \cdot \chi_{-}\right) \varphi_{-}+\left(\alpha_{a} \cdot \chi_{+}\right) \varphi_{+}+\sum_{i=a+2}^{r} k_{a i} \varphi_{i}
$$

\footnotetext{
${ }^{7}$ If we leave $\mathcal{G}_{0}^{0}$ unconstrained the resulting model belongs again to the non singular NA-Toda class of models [3],[6]. From the string theory point of view the class of singular NA Toda models are of great interest since they describe strings on specific black hole backgrounds.
} 
Now, if we consider Lie algebras whose Dynkin diagrams connect only nearest neighbours, i. e., $\Phi\left(\alpha_{a}\right)=\left(\alpha_{a} \cdot \chi_{-}\right) \varphi_{-}+\left(\alpha_{a} \cdot \chi_{+}\right) \varphi_{+}$, then the "no-torsion condition" implies $\Phi\left(\alpha_{a}\right)=0$. Considering case (iii), we have

$$
\alpha_{a} \cdot \chi_{-}=\alpha_{a} \cdot\left(\alpha_{a-1}+\alpha_{a}\right)=0, \quad \alpha_{a} \cdot \chi_{+}=\alpha_{a} \cdot\left(\alpha_{a+1}\right)=0 .
$$

In this case, the only solution for both equations is to take $a=r$ (in such a way that $\alpha_{r+1}=0$ ) and $\mathcal{G}=B_{r}$ (so that $\alpha_{r}^{2}=-\alpha_{r-1} \cdot \alpha_{r}=1$ ). This is precisely the case proposed by Leznov and Saveliev [25] and subsequently discussed by Gervais and Saveliev [20] and also by Bilal [21], for the particular case of $B_{2}$. For case (iv), the "no-torsion condition" requires that

$$
\alpha_{a-1} \cdot \alpha_{a}=0, \quad \alpha_{a} \cdot\left(\alpha_{a}+\alpha_{a+1}\right)=0
$$

Both are satisfied for $a=1, \mathcal{G}=C_{2}$, since $\alpha_{a-1}=0$ and also $\alpha_{1}^{2}=-\alpha_{1} \cdot \alpha_{2}=1$, respectively.

In general, the "no-torsion condition" (T-selfduality), i. e., $\Phi\left(\alpha_{a}\right)=0$, may be expressed in terms of the structure of the co-set $\mathcal{G}_{0} / \mathcal{G}_{0}^{0}=\frac{u(1)^{r-1} \otimes s l(2)}{u(1)}$. The crucial ingredient for the appearence of $\Phi\left(\alpha_{a}\right)$ arises from the conjugation

$$
\operatorname{Tr}\left(A_{0} g_{0}^{f} \bar{A}_{0}\left(g_{0}^{f}\right)^{-1}+A_{0} \bar{A}_{0}\right)=2 \lambda_{a}^{2}\left(1+\frac{2}{\alpha_{a}^{2}} \frac{\chi \psi \exp \left(\Phi\left(\alpha_{a}\right)\right)}{2 \lambda_{a}^{2}}\right) .
$$

Henceforth, if all generators belonging to the Cartan subalgebra parameterizing $g_{0}^{f}$ commute with $E_{ \pm \alpha_{a}}$, then $\Phi\left(\alpha_{a}\right)=0$, and therefore the structure of the co-set

$$
\frac{G_{0}}{G_{0}^{0}}=\frac{u(1)^{r-1} \otimes s l(2)}{u(1)}=u(1)^{r-1} \otimes \frac{s l(2)}{u(1)}
$$

is the general condition for the absence of the antisymmetric term in the action.

Summarizing, for finite Lie algebras, it was shown that the absence of the antisymmetric terms in the action of the axial NA - Toda models can only occur for $\mathcal{G}=B_{r}, a=r$ and $\epsilon_{ \pm}=\sum_{i=1}^{r-2} c_{ \pm i} E_{ \pm \alpha_{i}}+d_{ \pm} E_{ \pm\left(\alpha_{r}+\alpha_{r-1}\right)}$. In such case, $\mathcal{G}_{0}^{0}$ is generated by $Y \cdot H=\left(\frac{2 \lambda_{r}}{\alpha_{r}^{2}}-\frac{2 \lambda_{r-1}}{\alpha_{r-1}^{2}}\right) \cdot H$ and $\Phi(H)=\sum_{i=1}^{r-2} \varphi_{i} h_{i}+\varphi_{-}\left(\alpha_{r-1}+\alpha_{r}\right) \cdot H$. Due to the root structure of $B_{r}$, we verify that $\Phi\left(\alpha_{r}\right)=\alpha_{r} \cdot\left(\alpha_{r-1}+\alpha_{r}\right) \varphi_{-}=0$.

In order to extend the no torsion theorem (T-selfduality) to the case of infinite affine Lie algebras (i.e for the integrable perturbations of the conformal models of sect. 4.1) let we consider $h^{\prime}=1-\sum_{i \neq r} \frac{2}{\alpha_{i}^{2}} \lambda_{i} \cdot \alpha_{0}$, where $-\alpha_{0}$ is the highest root of $\mathcal{G}$ such that $\alpha_{0} \cdot\left(\frac{2 \lambda_{r}}{\alpha_{r}^{2}}-\frac{2 \lambda_{r-1}}{\alpha_{r-1}^{2}}\right)=0$ and the gradation $Q_{a}\left(h^{\prime}\right)$ that preserves the zero grade subalgebra $\mathcal{G}_{0}$, (apart from $\hat{c}$ and $\hat{d}$ ). We choose the cooresponding (affine) grade \pm elements in the form $\hat{\epsilon}_{+}=\epsilon_{+}+E_{\alpha_{0}}^{(1)}$. Since conformal and the affine models differ only by the potential term, the solution for the no torsion condition is also satisfied for infinite dimensional algebras, whose Dynkin diagram possess a $B_{r^{-}}$"tail like". An obvious solution is the untwisted $B_{r}^{(1)}$ model. Two new other solutions are given by the twisted affine Kac-Moody algebras $A_{2 r}^{(2)}$ and $D_{r+1}^{(2)}$ as we shall describe in detail in the next two subsections. 


\subsection{The $B_{r}^{(1)}$ Torsionless Affine NA Toda models}

In order to generalize the $B_{r}$ conformal models to the affine $B_{r}^{(1)}$ ones we take

$Q=2(r-1) \hat{d}+\sum_{i=1}^{r-1} \frac{2 \lambda_{i} \cdot H}{\alpha_{i}^{2}}$, which decomposes $B_{r}^{(1)}$ into graded subspaces. In particular, the zero grade subspace turns out to be $\mathcal{G}_{0}=S L(2) \otimes U(1)^{r-1}$, generated by $\left(E_{ \pm \alpha_{r}}^{(0)}, h_{1}, \cdots, h_{r}\right)$. Following the arguments of the conformal no torsion theorem of the sect. 4.1 , we have to choose

$$
\hat{\epsilon_{ \pm}}=\sum_{i=1}^{r-2} c_{ \pm i} E_{ \pm \alpha_{i}}^{(0)}+c_{ \pm(r-1)} E_{ \pm\left(\alpha_{r-1}+\alpha_{r}\right)}^{(0)}+c_{ \pm r} E_{ \pm \alpha_{0}}^{( \pm 1)}
$$

where $-\alpha_{0}$ (defined by $\left.\alpha_{0}+\alpha_{1}+2\left(\alpha_{2}+\cdots+\alpha_{r-1}+\alpha_{r}\right)=0\right)$ is the highest root of $B_{r}$ and $\mathcal{G}_{0}^{0}$ is generated by $Y \cdot H=\left(\frac{2 \lambda_{r}}{\alpha_{r}^{2}}-\frac{2 \lambda_{r-1}}{\alpha_{r-1}^{2}}\right) \cdot H$ ( such that $\left.\left[Y \cdot H, \hat{\epsilon_{ \pm}}\right]=0\right)$. The coset $\mathcal{G}_{0} / \mathcal{G}_{0}^{0}$ (with $\eta=0$ ) is then parametrized according to (2.13) with $\Phi(H)=\sum_{i=1}^{r-1} \mathcal{H}_{i} \varphi_{i}$, where $\mathcal{H}_{i}=\left(\alpha_{r}+\cdots \alpha_{i}\right) \cdot H$ so that $\operatorname{Tr}\left(\mathcal{H}_{i} \mathcal{H}_{j}\right)=\delta_{i j}, i, j=1, \cdots, r-1$ and the total effective action becomes

$$
S=-\frac{k}{4 \pi} \int d^{2} z\left(\frac{1}{4} \sum_{i=1}^{r-1} g^{\mu \nu} \partial_{\mu} \varphi_{i} \partial_{\nu} \varphi_{i}+g^{\mu \nu} \frac{\partial_{\mu} \psi \partial_{\nu} \chi}{1+\psi \chi}-2 V\right)
$$

The "affine potential" $\mathrm{V}$ for $(n>2)$ has the form

$$
V=\sum_{i=1}^{r-2}\left|c_{i}\right|^{2} e^{-\varphi_{i}+\varphi_{i+1}}+2\left|c_{r-1}\right|^{2}(1+2 \psi \chi) e^{-\varphi_{r-1}}+\left|c_{r}\right|^{2} e^{\varphi_{1}+\varphi_{2}}
$$

The case $r=2$, i.e. $\widehat{\mathcal{G}}=\widehat{S} O(5)$ has to be considered separately. Choosing now the grade \pm 1 constant elements as $\hat{\epsilon_{ \pm}}=E_{\alpha_{1}+\alpha_{2}}^{(0)}+E_{-\alpha_{1}-\alpha_{2}}^{(1)}$, denoting $\Phi\left(\alpha_{r-1}\right)=\varphi$ and by further changing the variables

$$
\psi \longrightarrow i \psi ; \quad \chi \longrightarrow i \psi^{*} ; \quad \varphi \longrightarrow i \varphi
$$

we derive the following real action

$$
S=-\frac{k}{4 \pi} \int d^{2} z\left(\frac{g^{\mu \nu} \partial_{\mu} \psi \partial_{\nu} \psi^{*}}{\left(1-\psi \psi^{*}\right)}+\frac{1}{4} g^{\mu \nu} \partial_{\mu} \varphi \partial_{\nu} \varphi+8\left(1-2 \psi \psi^{*}\right) \cos \varphi\right)
$$

\subsection{The twisted affine NA Toda Models}

The twisted affine Kac-Moody algebras are constructed from a finite dimensional algebra possessing a nontrivial symmetry of their Dynkin diagrams (this procedure is known as folding). Such symmetry can be extended to the algebra by an outer automorphism $\sigma$ [22], as

$$
\sigma\left(E_{\alpha}\right)=\eta_{\alpha} E_{\sigma(\alpha)}
$$

where $\eta_{\alpha}= \pm 1$. For the simple roots, $\eta_{\alpha_{i}}=1$. The signs can be consistently assigned to all generators since nonsimple roots can be written as sum of two other roots.

The no torsion theorem requires a $B_{r^{-}}$"tail like" structure which is fulfilled only by the $A_{2 r}^{(2)}$ and $D_{r+1}^{(2)}$ (see appendix N of ref. [22]). In both cases the automorphism is of order 2 
(i.e. $\sigma^{2}=1$ ). Let us denote by $\alpha$ the roots of the untwisted algebra $\mathcal{G}$. For the $A_{2 r}^{(2)}$ case, the automorphism is defined by

$$
\sigma\left(\alpha_{1}\right)=\alpha_{2 r}, \quad \sigma\left(\alpha_{2}\right)=\alpha_{2 r-1} \quad \cdots, \sigma\left(\alpha_{r-1}\right)=\alpha_{r}
$$

whilst for the $D_{r+1}^{(2)}$, the automorphism acts only in the "fish tail" of the Dynkin diagram of $D_{r+1}$, i.e.

$$
\sigma\left(E_{\alpha_{1}}\right)=E_{\alpha_{1}}, \cdots, \sigma\left(E_{\alpha_{r-1}}\right)=E_{\alpha_{r-1}}, \quad \sigma\left(E_{\alpha_{r}}\right)=E_{\alpha_{r+1}}
$$

The automorphism $\sigma$ defines a decomposition of the algebra $\mathcal{G}=\mathcal{G}_{\text {even }} \cup \mathcal{G}_{\text {odd }}$. The twisted affine algebra is then constructed from $\mathcal{G}$ assigning an affine index $m \in Z$ to the generators in $\mathcal{G}_{\text {even }}$ while $m \in Z+\frac{1}{2}$ to those in $\mathcal{G}_{\text {odd }}$ (see appendix $\mathrm{N}$ of [22]).

The simple root step operators for $A_{2 r}^{(2)}$ are

$$
E_{\beta_{i}}=E_{\alpha_{i}}^{(0)}+E_{\alpha_{2 r-i+1}}^{(0)}, \quad i=1, \cdots, r \quad E_{\beta_{0}}^{\left(\frac{1}{2}\right)}=E_{-\alpha_{1}-\cdots-\alpha_{2 r}}^{\left(\frac{1}{2}\right)}
$$

corresponding to the simple and highest roots

$$
\beta_{i}=\frac{1}{2}\left(\alpha_{i}+\alpha_{2 r-i+1}\right) i=1, \cdots, r, \quad-\alpha_{0}=\alpha_{1}+\cdots+\alpha_{2 r}=2\left(\beta_{1}+\cdots \beta_{r}\right)
$$

respectively. For $D_{r+1}^{(2)}$, the simple root step operators are

$$
\begin{aligned}
E_{\beta_{i}}=E_{\alpha_{i}}^{(0)}, i & =1, \cdots, r-1, \quad E_{\beta_{r}}=E_{\alpha_{r}}^{(0)}+E_{\alpha_{r+1}}^{(0)} \\
E_{\beta_{0}}^{\left(\frac{1}{2}\right)} & =E_{-\alpha_{1}-\cdots-\alpha_{r-1}-\alpha_{r+1}}^{\left(\frac{1}{2}\right)}-E_{-\alpha_{1}-\cdots-\alpha_{r-1}-\alpha_{r}}^{\left(\frac{1}{2}\right)}
\end{aligned}
$$

corresponding to the simple and highest roots

$\beta_{i}=\alpha_{i} \quad i=1, \cdots, r-1, \quad \beta_{r}=\frac{1}{2}\left(\alpha_{r}+\alpha_{r+1}\right), \quad-\alpha_{0}=\alpha_{1}+\cdots \alpha_{r-1}+\frac{1}{2}\left(\alpha_{r}+\alpha_{r+1}\right)=\beta_{1}+\cdots \beta_{r}$

where have denoted by $\beta$ the roots of the twisted (folded) algebra.

The corresponding torsionless affine NA Toda models are defined by introducing the following grading operators :

$$
Q_{A_{2 r}^{(2)}}=2(2 r-1) \hat{d}+\sum_{i \neq r, r+1}^{2 r} \frac{2 \lambda_{i} \cdot H}{\alpha_{i}^{2}}, \quad Q_{D_{r+1}^{(2)}}=(2 r-2) \hat{d}+\sum_{i=1}^{r} \frac{2 \lambda_{i} \cdot H}{\alpha_{i}^{2}}
$$

for $A_{2 r}^{(2)}$ and $D_{r+1}^{(2)}$ respectively, where $\lambda_{i}$ are the fundamental weights of the untwisted algebra $\mathcal{G}$, i.e. $\frac{2 \lambda_{i} \cdot \alpha_{j}}{\alpha_{j}^{2}}=\delta_{i j}$. Both models are specified by the constant grade \pm 1 operators $\hat{\epsilon}_{ \pm}$

$$
\hat{\epsilon}_{ \pm}=\sum_{i=1}^{r-2} c_{ \pm i} E_{ \pm \beta_{i}}+c_{ \pm(r-1)} E_{ \pm\left(\beta_{r-1}+\beta_{r}\right)}+c_{ \pm r} E_{\mp \beta_{0}}^{\left( \pm \frac{1}{2}\right)}
$$

where $\beta_{i}$ are the simple roots of the twisted affine algebra specified in (4.13) and in (4.15).

The grading operators (4.16) determine the zero grade subalgebra in both cases to be $\mathcal{G}_{0}=S L(2) \otimes U(1)^{r-1}$ generated by $E_{ \pm \beta_{r}}^{(0)}, h_{1}, \cdots, h_{r}$. Hence the zero grade subgroup is 
parametrized as in (4.2) with $\eta=0=\nu$. The factor group $\mathcal{G} / \mathcal{G}_{0}^{0}$ is given in (2.13), where $\mathcal{G}_{0}^{0}$ is generated by $Y \cdot H=\left(\frac{2 \mu_{r}}{\beta_{r}^{2}}-\frac{2 \mu_{r-1}}{\beta_{r-1}^{2}}\right) \cdot H$ and $\mu_{i}$ are the fundamental weights of the twisted algebra i.e. $\frac{2 \mu_{i} \cdot \beta_{j}}{\beta_{j}^{2}}=\delta_{i j}$. In order to decouple the $\varphi_{i}, \quad i=1, \cdots, r-1$ we choose an orthonormal basis for the Cartan subalgebra( i.e. $\left.\Phi(H)=\mathcal{H}_{i} \varphi_{i}\right)$ where

$$
\mathcal{H}_{i}=\left(\alpha_{i}+\cdots \alpha_{2 r-i+1}\right) \cdot H, \quad Y \cdot H=\mathcal{H}_{r}, \operatorname{Tr}\left(\mathcal{H}_{i} \mathcal{H}_{j}\right)=2 \delta_{i j}, i, j=1, \cdots r
$$

and

$$
\mathcal{H}_{i}=\left(\alpha_{r-i+1}+\cdots+\alpha_{r+1}\right) \cdot H, \quad Y \cdot H=\mathcal{H}_{r}, \operatorname{Tr}\left(\mathcal{H}_{i} \mathcal{H}_{j}\right)=\delta_{i j}, i, j=1, \cdots r
$$

for $A_{2 r}^{(2)}$ and $D_{r+1}^{(2)}$ respectively.

The Lagrangeans of the corresponding (axial gauged IMs), based on the twisted affine algebras in consideration, are obtained from (2.16) (with $\eta=0$ ) and the $\epsilon_{ \pm}$given by (4.17). Up to a multiplicative factor $-\frac{k}{2 \pi}$ they are given by

$$
\mathcal{L}_{A_{2 r}^{(2)}}=\frac{\partial \chi \bar{\partial} \psi}{1+\frac{1}{2} \psi \chi}+\frac{1}{2} \sum_{i=1}^{r-1} \partial \varphi_{i} \bar{\partial} \varphi_{i}-V_{A_{2 r}^{(2)}}
$$

and

$$
\mathcal{L}_{D_{r+1}^{(2)}}=2 \frac{\partial \chi \bar{\partial} \psi}{1+\psi \chi}+\frac{1}{2} \sum_{i=1}^{r-1} \partial \varphi_{i} \bar{\partial} \varphi_{i}-V_{D_{r+1}^{(2)}}
$$

The potentials of these twisted affine singular NA - Toda models have the form

$$
V_{A_{2 r}^{(2)}}=\sum_{i=1}^{r-2}\left|c_{i}\right|^{2} e^{-\varphi_{i}+\varphi_{i+1}}+\frac{1}{2}\left|c_{r}\right|^{2} e^{2 \varphi_{1}}+\left|c_{r-1}\right|^{2} e^{-\varphi_{r-1}}(1+\psi \chi)
$$

and

$$
V_{D_{r+1}^{(2)}}=\sum_{i=1}^{r-2}\left|c_{i}\right|^{2} e^{-\varphi_{i}+\varphi_{i+1}}+\frac{1}{2}\left|c_{r}\right|^{2} e^{\varphi_{1}}+\left|c_{r-1}\right|^{2} e^{-\varphi_{r-1}}(1+2 \psi \chi)
$$

The T -selfdual models described by (4.6), (4.20) and (4.21) turns out to coincide with those proposed by Fateev in [14].

\section{Zero Curvature}

The equations of motion for the NA Toda models are known to be of the form 25]

$$
\bar{\partial}\left(B^{-1} \partial B\right)+\left[\hat{\epsilon_{-}}, B^{-1} \hat{\epsilon_{+}} B\right]=0, \quad \partial\left(\bar{\partial} B B^{-1}\right)-\left[\hat{\epsilon_{+}}, B \hat{\epsilon_{-}} B^{-1}\right]=0
$$

The subsidiary constraint $J_{Y \cdot H}=\operatorname{Tr}\left(B^{-1} \partial B Y \cdot H\right)=\bar{J}_{Y \cdot H}=\operatorname{Tr}\left(\bar{\partial} B B^{-1} Y \cdot H\right)=0$ can be consistenly imposed since $\left[Y \cdot H, \hat{\epsilon_{ \pm}}\right]=0$ (as can be seen from (5.1) by taking the trace with $Y . H$ ). We next consider the axial models only. As we have mentioned in Sect.3 the vector models Lagrangeans (and equations of motion) can be obtained from the axial ones 
by the nonlocal change of the fields (see for example eqs.(3.9) and (3.10) for the $A_{r}^{(1)}$ and $B_{r}^{(1)}$ models). Solving those equations for the nonlocal field $R$ yields

$$
\partial R=\left(\frac{Y \cdot \alpha_{r}}{Y^{2}}\right) \frac{\psi \partial \chi}{\Delta} e^{\Phi\left(\alpha_{r}\right)}, \quad \bar{\partial} R=\left(\frac{Y \cdot \alpha_{r}}{Y^{2}}\right) \frac{\chi \bar{\partial} \psi}{\Delta} e^{\Phi\left(\alpha_{r}\right)}
$$

The equations of motion for the fields $\psi, \chi$ and $\varphi_{i}, i=1, \cdots, r-1$ (obtained from (5.1) by imposing the constraints (5.2)) coincide precisely with the Euler-Lagrange equations derived from (4.20) and (4.21). Alternatively, (5.1) admits a zero curvature representation $\partial \bar{A}-\bar{\partial} A+[A, \bar{A}]=0$, where

$$
A=\hat{\epsilon_{-}}+B^{-1} \partial B, \quad \bar{A}=-B^{-1} \hat{\epsilon_{+}} B
$$

Whenever the constraints (5.2) are incorporated into $A$ and $\bar{A}$ in (5.3), equations (5.1) yields the zero curvature representation of the affine singular NA -Toda models. Such argument is valid for all conformal, affine and conformal affine NA - Toda models, in particular for the torsionless class of models discussed in the previous section.

Using the explicit parametrization of $B$ given in (2.11), (with $\eta=0$ ) the corresponding $\hat{\epsilon_{ \pm}}$specified in (4.17), (4.13) and (4.15) together with (5.2) (with $Y$ given by eqs. (4.18) and (4.19)), we obtain the flat connections $A$ and $\bar{A}$ in the following form :

(a) the $A_{2 r}^{(2)}$ affine NA - Toda model

$$
\begin{aligned}
A_{A_{2 r}^{(2)}} & =\sum_{i=1}^{r-2} c_{i}\left(E_{-\alpha_{i}}^{(0)}+E_{-\alpha_{2 r-i+1}}^{(0)}\right)+c_{r-1}\left(E_{-\alpha_{r}-\alpha_{r-1}}^{(0)}+E_{-\alpha_{r+1}-\alpha_{r+2}}^{(0)}\right) \\
& +c_{r} E_{\alpha_{1}+\cdots+\alpha_{2 r}}^{\left(-\frac{1}{2}\right)}+\partial \psi e^{-\frac{1}{2} R}\left(E_{\alpha_{r}}^{(0)}+E_{\alpha_{r+1}}^{(0)}\right)+\sum_{i=1}^{r-1} \partial \varphi_{i} \mathcal{H}_{i} \\
& +\frac{\partial \chi}{\Delta} e^{\frac{1}{2} R}\left(E_{-\alpha_{r}}^{(0)}+E_{-\alpha_{r+1}}^{(0)}\right)
\end{aligned}
$$

and

$$
\begin{aligned}
-\bar{A}_{A_{2 r}}^{(2)} & =\sum_{i=1}^{r-2} c_{i} e^{-\varphi_{i}+\varphi_{i+1}}\left(E_{\alpha_{i}}^{(0)}+E_{\alpha_{2 r-i+1}}^{(0)}\right)+c_{r} e^{2 \varphi_{1}} E_{-\alpha_{1}-\cdots-\alpha_{2 r}}^{\left(\frac{1}{2}\right)} \\
& +c_{r-1} e^{-\varphi_{r-1}}\left(E_{\alpha_{r}+\alpha_{r-1}}^{(0)}+E_{\alpha_{r+1}+\alpha_{r+2}}^{(0)}\right) \\
& +c_{r-1} \psi e^{-\frac{1}{2} R-\varphi_{r-1}}\left(E_{\alpha_{r+1}+\alpha_{r}+\alpha_{r-1}}^{(0)}-E_{\alpha_{r}+\alpha_{r+1}+\alpha_{r+2}}^{(0)}\right) \\
& +c_{r-1} \chi e^{\frac{1}{2} R-\varphi_{r-1}}\left(E_{\alpha_{r-1}}^{(0)}-E_{\alpha_{r+2}}^{(0)}\right)+c_{r-1} \psi \chi e^{-\varphi_{r-1}}\left(E_{\alpha_{r}+\alpha_{r-1}}^{(0)}-E_{\alpha_{r+1}+\alpha_{r+2}}^{(0)}\right) \\
& +\frac{1}{2} c_{r-1} \psi^{2} \chi e^{-\varphi_{r-1}-\frac{1}{2} R}\left(E_{\alpha_{r+1}+\alpha_{r}+\alpha_{r-1}}^{(0)}-E_{\alpha_{r}+\alpha_{r+1}+\alpha_{r+2}}^{(0)}\right)
\end{aligned}
$$

(b) the $D_{r+1}^{(2)}$ affine NA - Toda model

$$
\begin{aligned}
A_{D_{r+1}^{(2)}} & =\sum_{i=1}^{r-2} c_{i} E_{-\alpha_{i}}^{(0)}+c_{r-1}\left(E_{-\alpha_{r}-\alpha_{r-1}}^{(0)}+E_{-\alpha_{r-1}-\alpha_{r+1}}^{(0)}\right) \\
& +c_{r}\left(E_{\left(\alpha_{1}+\cdots+\alpha_{r-1}+\alpha_{r+1}\right)}^{\left(-\frac{1}{2}\right)}-E_{\left(\alpha_{1}+\cdots+\alpha_{r}+\alpha_{r+1}\right)}^{\left(-\frac{1}{2}\right)}\right)+\partial \psi e^{-\frac{1}{2} R}\left(E_{\alpha_{r}}^{(0)}+E_{\alpha_{r+1}}^{(0)}\right) \\
& +\sum_{i=1}^{r-1} \partial \varphi_{i} \mathcal{H}_{i}+\frac{\partial \chi}{\Delta} e^{\frac{1}{2} R}\left(E_{-\alpha_{r}}^{(0)}+E_{-\alpha_{r+1}}^{(0)}\right)
\end{aligned}
$$


and

$$
\begin{aligned}
-\bar{A}_{D_{r+1}^{(2)}}^{(2)} & =\sum_{i=1}^{r-2} c_{i} e^{-\varphi_{i}+\varphi_{i+1}} E_{\alpha_{i}}^{(0)}+c_{r-1} e^{-\varphi_{r-1}}\left(E_{\alpha_{r}+\alpha_{r-1}}^{(0)}+E_{\alpha_{r+1}+\alpha_{r-1}}^{(0)}\right) \\
& +2 c_{r-1} \psi e^{-\frac{1}{2} R-\varphi_{r-1}} E_{\alpha_{r+1}+\alpha_{r}+\alpha_{r-1}}^{(0)}+2 c_{r-1} \chi e^{\frac{1}{2} R-\varphi_{r-1}} E_{\alpha_{r-1}}^{(0)} \\
& +2 c_{r-1} \psi \chi e^{-\varphi_{r-1}}\left(E_{\alpha_{r+1}+\alpha_{r-1}}^{(0)}+E_{\alpha_{r-1}+\alpha_{r}}^{(0)}\right)+c_{r-1} \psi^{2} \chi e^{-\frac{1}{2} R-\varphi_{r-1}} E_{\alpha_{r+1}+\alpha_{r}+\alpha_{r-1}}^{(0)} \\
& +c_{r+1} e^{\varphi_{1}}\left(E_{-\left(\alpha_{1}+\cdots+\alpha_{r-1}+\alpha_{r+1}\right)}^{\left(\frac{1}{2}\right)}-E_{-\left(\alpha_{1}+\cdots+\alpha_{r}+\alpha_{r+1}\right)}^{\left(\frac{1}{2}\right)}\right)
\end{aligned}
$$

For the untwisted affine $B_{r}^{(1)}$ model of the previous section the zero curvature representation is obtained from the following flat connections :

$$
\begin{gathered}
A_{B_{r}^{(1)}}=\sum_{i=1}^{r-2} c_{i} E_{-\alpha_{i}}^{(0)}+c_{r-1} E_{-\alpha_{r}-\alpha_{r-1}}^{(0)}+c_{r} E_{\alpha_{1}+2\left(\alpha_{2}+\cdots+\alpha_{r}\right)}^{(-1)} \\
+\partial \psi e^{-\frac{1}{2} R} E_{\alpha_{r}}^{(0)}+\sum_{i=1}^{r-1} \partial \varphi_{i} \mathcal{H}_{i}+\frac{\partial \chi}{\Delta} e^{\frac{1}{2} R} E_{-\alpha_{r}}^{(0)} \\
-\bar{A}_{B_{r}^{(1)}}=\sum_{i=1}^{r-2} c_{i} e^{-\varphi_{i}+\varphi_{i+1}} E_{\alpha_{i}}^{(0)}+c_{r} e^{\varphi_{1}+\varphi_{2}} E_{-\left(\alpha_{1}+2\left(\alpha_{2}+\cdots+\alpha_{r}\right)\right)}^{(1)}+2 \chi e^{\varphi_{r-1}+\frac{1}{2} R} E_{\alpha_{r-1}}^{(0)} \\
+c_{r-1}(1+2 \psi \chi) e^{-\varphi_{r-1}} E_{\alpha_{r-1}+\alpha_{r}}^{(0)}-2 c_{r-1} e^{-\varphi_{r-1}-\frac{1}{2} R} \psi(1+\psi \chi) E_{\alpha_{r-1}+2 \alpha_{r}}^{(0)}
\end{gathered}
$$

The zero curvature representation of the subclass of torsionless singular affine NA - Toda models shows that they are in fact classically integrable field theories. The construction of the previous sections provides a systematic affine Lie algebraic structure underlying those models, which is known to play the crucial role in the construction of their finite energy soliton solutions.

\section{Conclusions}

We have constructed a class of affine (and conformal affine) NA-Toda models from the gauged two-loop WZNW models, in which left and right symmetries are incorporated by a suitable choice of grading operator $Q$ and of grade \pm 1 constant generators $\epsilon_{ \pm}$. We have shown that for non abelian zero grade subalgebra $\mathcal{G}_{0}$, it is possible to reduce even further the phase space by constraining to zero the currents associated to generators $Y \cdot H$, commuting with $\left.\epsilon_{ \pm}\left(Y \cdot H \in \mathcal{G}_{0}^{0}\right)\right)$. There exists two inequivalent manners to gauge fix $\mathcal{G}_{0}^{0}=U(1)$-the axial and the vector gaugings. Similarly to the T-duality transformations between the axial and vector gauged $\mathrm{G} / \mathrm{H}$ - WZNW models, one can find the off-critical counterpart of the conformal T-duality, relating now the axial and vector families of IM's constructed in Sect. 2 . We further analize the problem of deriving the Lie algebraic condition which defines a class of T-selfdual torsionless models, for the case $\mathcal{G}_{0}^{0}=U(1)$. The action for those models were systematicaly constructed and shown to coincide with those proposed by Fateev [14], 
describing the strong coupling limit of specific 2-d models representing the complex sineGordon (i.e. Lund-Regge [13]) interacting with Toda-like models. Their weak coupling limit appears to be the Thirring model coupled to certain affine Toda theories [14].

As we have mentioned in sects. 1 and 3 the conformal $\sigma$-model limits of the axial and vector dyonic IM's describe critical $D=r+1$ strings on black hole backgrounds [7]. One may wonder whether the nonconformal (i.e. off-critical) dyonic IM's (representing integrable perturbations of these string models ) have some string field theory applications. As is well known [26] the relation between 2-d conformal model and its integrable perturbations allows to describe the off-critical behaviour of the original conformal model as well as the RG flow from ultraviolet to infrared (in the case of unitary CFT's ) see [27], [8] and references therein. Hence the properties of the admissible integrable perturbations of the conformal $\sigma$-models are an important ingredient in the description of the space of string backgrounds (satisfying certain low energy physical requirements). As it advocated by B. Zwiebach 28 the nonconformal versions of the conformal string backgrounds appears to be the main tool for the construction of the off-shell string field theory (SFT). Among all the possible nonconformal backgrounds the integrable ones (admiting "worldsheet" soliton solutions) have the advantage to offer powerfull methods for studying the string S-duality.

The dyonic integrable models studied in the present paper represent the simplest family of IM's with one U(1) global symmetry. Translated to the string language this means that the target-space metrics $\left(E_{M N}(X), \phi(X), T(X)\right)$ are independent of one of the coordinates $X_{M}\left(\theta=\frac{1}{2} \ln \frac{\chi}{\psi}\right.$ in our case $)$ and that the relevant operator does not break this isometry. Models with more isometries (generic abelian T-duality) or those admiting isotropies ( nonabelian T-duality) appears to be useful for the construction of physically intersting string models $([10])$. The problem of construction of their integrable perturbations requires to consider more general affine $\hat{\mathcal{G}}_{r}$-NA Toda models defined by grading operators as for example $Q_{a, b, \cdots}=h_{a, b, \cdots} \hat{d}+\sum_{i \neq a, b \cdots}^{n} \frac{2 \lambda_{i} \cdot H}{\alpha_{i}^{2}}$ and appropriately choosen $\epsilon_{ \pm}$. They should allow larger(than $\mathrm{U}(1)$ ) invariant subgroup $\mathcal{G}_{0}^{0}$ (non-abelian in general). An important characteristic of such IM's is that their physical fields belongs to , say $\mathcal{G}_{0} / \mathcal{G}_{0}^{0}=\frac{S L(2) \otimes U(1)^{r-1}}{U(1)^{s}}$,

$s=2, \cdots r-1$ (i.e with s isometries), or $\mathcal{G}_{0} / \mathcal{G}_{0}^{0}=\frac{S L(2) \otimes S L(2) \otimes U(1)^{r-2}}{U(1)^{s}}$, or $\mathcal{G}_{0} / \mathcal{G}_{0}^{0}=\frac{S L(3) \otimes U(1)^{r-2}}{U(2)}$ (the corresponding IM's represent string backgrounds with one isometry and SL(2) as isotropy group ), etc. The methods needed in the construction of such IM's and for the investigation of their T-duality properties appears to be straightforward generalization of the methods we have developed for the simplest case of $\mathcal{G}_{0}^{0}=U(1)$. An intersting open problem is the classification of the affine NA-Toda IM's according to the number of the physical fields ( i.e. the dimension of the string target space ) and their symmetry groups $\mathcal{G}_{0}^{0}$ ( i.e. the symmetries of the string backgrounds). We hope that the simplest dyonic IM's studied in the present paper and the methods we have developed might contribute to the complete description of the space of the integrable (nonconformal) string backgrounds.

Acknowledgments We are grateful to CNPq, FAPESP and UNESP for financial support.

\section{References}


[1] L.D. Faddeev and L. Takhtajan, "Hamiltonian methods in the Theory of Solitons", (Springer, Berlin ),1987

[2] A. N. Leznov, M. V. Saveliev, Group Theoretical Methods for Integration of Nonlinear Dynamical Systems, Progress in Physics, Vol. 15 (1992), Birkhauser Verlag, Berlin

[3] J. Balog, L. Feher and L. O'Raifeartaigh, Ann. of Phys. 303, (1990), 76-136

[4] L.A. Ferreira, J.F. Gomes, A. Schwimmer and A.H. Zimerman, Phys. Lett. 274B (1992) 65

[5] H. Aratyn, L.A. Ferreira, J.F. Gomes and A.H. Zimerman, Phys. Lett B 254 (1991) 372

[6] L.A. Ferreira, J.L. Miramontes and J.S. Guillen, Nucl. Phys. B449 (1995);

C. R. Fernandez-Pousa, M. V. Gallas, T. J. Hollowood and J.L. Miramontes Nucl.Phys. B484 (1997),609

[7] J.H. Horne and G.T. Horowitz, Nucl. Phys. B368 (1992) 444, P. Ginsparg and F. Quevedo, Nucl. Phys. B385 (1992) 527

[8] A.A. Tseytlin, Nucl. Phys. B399 (1993) 601; Nucl. Phys. B411 (1994) 509

[9] J.F. Gomes, E.P. Gueuvoghlanian, G.M. Sotkov and A.H. Zimerman, IFT-Unesp preprint 064/2000, "Electrically Charged Topological Solitons", hepth/0007169; see also "Dyonic Integrable models", hepth/0011187

[10] A. Giveon, M. Porrati and E. Rabinovici, Phys. Reports 244 (1994) 77

[11] E. Alvarez, L. Alvarez-Gaume, J.L.F. Barbon and Y. Lozano Nucl. Phys. B415 (1994) 71, hepth/9309039

[12] O. Alvarez and Chien-Hao Liu, Commun. Math. Phys. 179 (1996) 185, hepth/9503226

[13] F. Lund, Ann. of Phys. 415 (1978) 251;

F. Lund and T. Regge, Phys. Rev. D14 (1976) 1524

[14] V.A. Fateev, Nucl. Phys. B479 (1996) 594

[15] K. Gawedzki and A. Kupiainen, Nucl. Phys. B320 (1989) 625

[16] J.F. Gomes, F.E.M. da Silveira, G.M. Sotkov and A.H. Zimerman, "Singular NonAbelian Toda Theories", hepth 9810057, also in "Nonassociative Algebras and its Applications", Lec. Notes in Mathematics, Ed. R. Costa, et. al., Marcel Dekker, (2000), p. 125-136; J.F. Gomes, E. P. Gueuvoghlanian, F.E.M. da Silveira, G.M. Sotkov and A.H. Zimerman, "Singular Conformal, and Conformal Affine Non-Abelian Toda Theories", M.V. Saveliev Memorial Volume, Dubna (1999), p. 38-50, Ed. A.N. Sissakian.

[17] J.F. Gomes, G.M. Sotkov and A.H. Zimerman, " $S L(2, R)_{q}$ Symmetries of Non-Abelian Toda theories" hepth/9803122, Phys. Lett. 435B (1998) 49 
[18] J.F. Gomes, G.M. Sotkov and A.H. Zimerman, "Non Abelian Toda Theories From Parafermionic reductions of WZNW model", hepth/9803234, Ann. of Phys.274, (1999), 289-362

[19] E. Witten, Phys. Rev. Lett. 38 (1978) 121

[20] J.-L. Gervais and M. V. Saveliev, Phys. Lett. 286B (1992) 271

[21] A. Bilal, Nucl. Phys. B422 (1994) 258

[22] J.F. Cornwell, Group Theory in Physics, Vol. 3, Academic Press (1989)

[23] E. Alvarez, L. Alvarez-Gaumè and Y. Lozano, Nucl. Phys. Proc. Suppl. 41(1995)1

[24] T.Busher, Phys. Lett. 159B (1985) 127, Phys. Lett. 194B (1987) 59, Phys. Lett. 201B (1988) 466

[25] A. N. Leznov and M. V. Saveliev, Commun. Math. Phys. 89 (1983) 59

[26] A.B. Zamolodchikov, JETP Lett. 43,(1986)565; Sov. J. Nucl. Phys. 46 (1987)1090; T. Banks and E. Martinec, Nucl. Phys. B294 (1987) 733

[27] P. Haagensen, " Duality and the Renormalization Group", MIT-CTP-2668, hepth/9708110

[28] B. Zwiebach, Nucl. Phys. B480 (1996) 541 